\title{
Vaccination obligatoire des travailleurs de la santé : analyse juridique et politique
}

\author{
Colleen M. Flood JSD, Bryan Thomas JSD, Kumanan Wilson MD
}

Citation : CMAJ 2021 February 8;193:E217-20. doi : 10.1503/cmaj.202755-f; diffusion hâtive le 19 janvier 2021

Voir la version anglaise de l'article ici : www.cmaj.ca/lookup/doi/10.1503/cmaj.202755

M

aintenant que des vaccins contre le coronavirus du syndrome respiratoire aigu sévère 2 (SRAS-CoV-2) ont été approuvés, la vaccination des travailleurs de la santé du Canada qui sont en contact direct avec des patients est une priorité ${ }^{1}$. La question devient la suivante : les gouvernements, les organismes de santé publics et les entrepreneurs privés, comme les propriétaires et administrateurs d'établissements de soins de longue durée, devraient-ils faire le choix controversé de rendre la vaccination contre le SRASCoV-2 obligatoire, que ce soit par réglementation directe ou dans le cadre des conditions d'emploi? Une telle décision pourrait se justifier parce que la vaccination empêcherait directement les travailleurs et les patients dont ils prennent soin de contracter le virus. La prévention de la maladie à coronavirus 2019 (COVID-19) chez les personnes vaccinées éviterait également l'épuisement excessif du personnel de la santé. Notons toutefois qu'il est possible que les politiques encadrant la vaccination soient contestées. Par le passé, le droit du travail a été invoqué pour contester des politiques de vaccination obligatoire contre la grippe, et on pourrait en théorie présenter une contestation aux termes des lois sur les droits de la personne ou de la Charte canadienne des droits et libertés ${ }^{2}$. Nous discuterons ici de la jurisprudence issue de tentatives passées de rendre le vaccin contre la grippe obligatoire pour les travailleurs de la santé et de son applicabilité au contexte de la vaccination contre le SRAS-CoV-2, et proposerons des arguments juridiques que les gouvernements et les employeurs (p. ex. : hôpitaux publics et établissements de soins de longue durée) pourront avancer pour justifier la vaccination obligatoire.

\section{La jurisprudence sur la vaccination contre la grippe s'applique-t-elle à celle contre le SRAS-CoV-2?}

La majorité de la jurisprudence en lien avec la vaccination des travailleurs de la santé contre la grippe provient de décisions d'arbitres du travail (et non de tribunaux) réglant des différends entre des employeurs (p. ex. : hôpitaux publics) et des syndicats

\section{POINTS CLÉS}

- L'offre d'un vaccin efficace à tous les travailleurs de la santé au Canada protégerait à la fois l'effectif et les patients, réduisant du coup le fardeau total de la maladie à coronavirus 2019 sur les services et assurant la présence d'un personnel suffisant pour répondre aux besoins durant la pandémie.

- Les gouvernements provinciaux devraient mettre en place une réglementation encadrant la vaccination obligatoire des travailleurs de la santé s'appliquant à la fois aux secteurs public et privé, plutôt que de laisser ce choix à la discrétion des employeurs individuels.

- Si certains employeurs décidaient d'imposer la vaccination à leur personnel, la légalité de ce mandat serait vraisemblablement déterminée par les lois du travail encadrant le caractère raisonnable de la directive de l'employeur, comme en témoigne la jurisprudence relative à la vaccination obligatoire contre le virus de la grippe.

- Un mandat gouvernemental imposant la vaccination des travailleurs de la santé peut être contesté en vertu de la Charte canadienne des droits et libertés, mais, considérant l'état actuel des connaissances, il est probable qu'une telle contestation n'ait pas gain de cause si des exceptions ont été faites pour les personnes ne pouvant pas recevoir le vaccin en raison de problèmes de santé sous-jacents ou pour celles qui s'opposent à la vaccination pour des raisons religieuses ou pour objection de conscience.

- Les opposants pourraient avancer que les travailleurs de la santé ont le droit de porter de l'équipement de protection individuelle (EPI) au lieu de se faire vacciner, et donc que les gouvernements doivent appuyer la surveillance vaccinale et se tenir au courant des derniers développements concernant l'efficacité et l'innocuité des divers vaccins contre le coronavirus du syndrome respiratoire aigu sévère 2 (SRAS-CoV-2) comparativement à l'efficacité de l'EPI dans la réduction de la transmission du SRAS-CoV-2.

de travailleurs de la santé. Les ententes conclues en contexte de droit du travail ne limitent pas les options des gouvernements canadiens ou des employeurs concernant la vaccination contre le SRAS-CoV-2. Par exemple, une entente conclue entre le gouvernement et les infirmières de la Colombie-Britannique en décembre 2019 qui donnait à chaque infirmière le droit de se 
faire vacciner ou non contre la grippe ne signifie pas qu'à l'avenir, la capacité du gouvernement de la ColombieBritannique, des hôpitaux publics et des établissements de soins de longue durée d'imposer la vaccination contre le SRASCoV-2 est elle aussi limitée. De plus, la jurisprudence établie dans le contexte de la vaccination contre la grippe ne pourra pas être appliquée automatiquement au contexte de la COVID19 : le droit doit s'adapter à l'évolution des connaissances scientifiques. Exemple pertinent : si on le compare aux diverses souches de grippe, le SRAS-CoV-2 est plus facilement transmissible et a un taux de létalité plus élevé ${ }^{3,4}$.

Le développement relativement accéléré des vaccins contre le SRAS-CoV-2 (moins d'un an) doit également être pris en compte : certains pourraient s'inquiéter davantage de la fiabilité des données sur l'efficacité et l'innocuité des vaccins. Il ne faut toutefois pas oublier que le vaccin contre la grippe est différent chaque année $e^{5}$. Au moment de rédiger cet article, on ne savait pas encore si les nouveaux vaccins prévenaient la transmission de tous les variants du virus ni si les résultats des essais seraient reproduits parfaitement en contexte réel. De nouvelles données probantes sur l'innocuité, l'efficacité, la réduction de l'infectivité et la durée de l'immunité de chaque vaccin seront publiées au fil du déploiement des programmes de vaccination. Tous ces facteurs devront être pris en compte au moment d'évaluer les questions juridiques. Comme on dispose actuellement de données limitées sur la capacité des programmes de vaccination à prévenir la transmission, on est en droit de se demander si d'autres mesures - le port du masque, par exemple - pourraient avoir une efficacité suffisante pour rendre inutile l'imposition de la vaccination.

\section{Quelles lois s'appliquent à la vaccination obligatoire des travailleurs de la santé?}

D'un point de vue juridique, l'établissement d'exigences pour les travailleurs de la santé dépend en partie des décideurs en cause (encadré 1). Par exemple, il est important de savoir si les politiques ont été imposées par l'employeur (p. ex. : hôpitaux ou établissements de soins de longue durée) ou directement par les gouvernements. En effet, les actions de ses derniers ouvrent la porte à une contestation en vertu de la Charte canadienne des droits et libertés, par exemple en invoquant l'article 7, qui garantit le droit à la vie, à la liberté et à la sécurité de la personne. Cela dit, la Charte s'applique beaucoup plus rarement aux organismes non gouvernementaux - seulement dans les cas où ils agissent « en gouvernements » (en appliquant directement une politique gouvernementale). La Charte ne s'appliquerait donc pas à un établissement privé de soins de longue durée décidant de sa propre initiative de rendre obligatoire la vaccination des travailleurs de la santé, mais le mandat de l'employeur pourrait être contesté en vertu d'une loi s'appliquant au travail. Par contre, si un gouvernement provincial oblige tous les travailleurs de la santé à se faire vacciner et qu'un établissement privé choisit d'appliquer cette obligation à ces employés, une contestation en vertu de la Charte est possible (mais probablement vouée à l'échec, comme on l'expliquera plus loin).
À notre avis, les gouvernements provinciaux ne devraient pas laisser la décision de rendre la vaccination obligatoire ou non aux choix individuels - non uniformes - des différents employeurs; ils devraient plutôt établir des règles claires pour tous les milieux de travail publics et privés. En effet, la gravité des conséquences de ne pas étendre la portée de règles provinciales sur le contrôle des infections aux organismes privés comme les établissements de soins de longue durée est devenue évidente durant la première vague de la pandémie de COVID-19. Il sera impératif de ne pas répéter cette erreur avec les exigences de vaccination contre le SRAS-CoV-2.

\section{Contestation du mandat de l'employeur de se faire vacciner ou de porter un masque en vertu des lois du travail}

La plupart des contestations judiciaires de la vaccination obligatoire des travailleurs de la santé contre la grippe sont issues du contexte du droit du travail - plus précisément, des tribunaux arbitraux -; ces décisions ont une valeur de précédent limitée dans le contexte d'une hypothétique obligation par un gouvernement canadien de faire vacciner les travailleurs de la santé contre le SRAS-CoV-2. Cela dit, ces décisions pourraient devenir pertinentes si les gouvernements renoncent à promulguer des règles claires pour tous les contextes, contrairement à ce que nous recommandons plus bas, et décident plutôt de laisser la décision à la discrétion de chaque employeur. Dans ce dernier cas, il s'agirait plutôt de déterminer si une telle obligation constitue un exercice raisonnable des droits de gestion.

Le droit du travail se concentre actuellement sur l'argument que d'obliger un employé à choisir entre se faire vacciner ou se faire imposer un retrait du travail (sans salaire), ou entre se faire vacciner ou porter un masque, constituent un exercice unilatéral déraisonnable des droits de l'employeur. Dans leur argumentaire concernant le caractère déraisonnable, les syndicats se sont basés sur la science, contestant l'efficacité des masques et faisant valoir que la transmission de la grippe par des porteurs asymptomatiques est peu probable (un argument qui ne tiendrait probablement pas la route dans le cas du SRAS-CoV-2). Les syndicats ont également étayé leur position en avançant que la vaccination obligatoire contre la grippe viole les droits garantis par la Charte (droits à la liberté et à la sécurité de la personne, article 7 ) et les lois sur la protection de la vie privée et porte atteinte aux droits de la personne (ex. : protection contre la discrimination basée sur un handicap physique) même lorsqu'il n'est pas évident que la Charte s'appliquerait aux actions de l'établissement de santé.

Le traitement d'une contestation de la vaccination obligatoire en vertu du droit du travail peut aussi dépendre de la force de l'obligation. Par exemple, dans St. Peter's Health System v. CUPE, Local 778, l'employeur avait catégoriquement imposé la vaccination comme condition de travail durant une éclosion de grippe, et exigé le retrait sans salaire des employés refusant la vaccination. Après un vaste examen des précédents 


\section{Lois s'appliquant au travail}

- L'exigence de vaccination découle-t-elle d'un acte du gouvernement (p. ex. : un arrêté d'un ministre de la Santé)? Si oui, prendre en compte le contexte de la Charte (colonne de droite).

- L'exigence de vaccination est-elle raisonnable?

- Quels sont les effets négatifs potentiels de la COVID-19?

- Quelles sont les preuves de l'efficacité des vaccins contre ces effets négatifs?

- Quelles sont les preuves que les vaccins posent un risque pour les travailleurs de la santé?

- Les travailleurs de la santé pourraient-ils avoir l'option de porter de l'EPI à la place?

- Ya-t-il des preuves que les vaccins sont plus efficaces que l'EPI?

- Y a-t-il des considérations pragmatiques (p. ex. : coût, pénuries, difficulté d'application) qui font du recours à l'EPI une option déraisonnable?

\section{Contexte de la Charte canadienne des droits et libertés}

- Existe-t-il un acte du gouvernement (p. ex. : arrêté, loi, règlement) obligeant les travailleurs de la santé à se faire vacciner?

- L'arrêté impose-t-il un traitement médical forcé (mettant en jeu le droit à la liberté ou à la sécurité de la personne de la Charte)?

- Les conséquences d'un refus sont-elles suffisamment graves pour rendre le choix caduc?

- Les précédents juridiques de la Charte indiquent fortement que les "droits économiques », ce qui inclut le droit de pratiquer une profession, ne sont pas protégés.

- La portée de l'exigence de vaccination est-elle trop grande?

- Une politique plus permissive de style « vaccin ou EPI » permettraitelle au gouvernement d'atteindre son objectif?

- Ya-t-il des considérations pragmatiques (p. ex. : coût, pénuries, difficulté d'application) qui font du recours à l'EPI une option déraisonnable?

- La politique pourrait-elle être calibrée plus soigneusement (p. ex. : imposée seulement aux établissements ayant déjà connu des éclosions)?

Remarque : COVID-19 = maladie à coronavirus 2019, EPI = équipement de protection individuelle.

dans le contexte de la Charte et de la common law, l'arbitre a conclu que la politique portait atteinte au droit à la sécurité de la personne - « [traduction] le traitement médical forcé... constitue une agression s'il y a absence de consentement ${ }^{6}$ »et a jugé la politique inapplicable. Des juristes analysant les tendances globales dans le domaine ont affirmé que St. Peter's est une exception, puisque dans la plupart des cas, les arbitres ont conclu que les politiques interdisant aux travailleurs de la santé non vaccinés de se présenter au travail durant une éclosion dans leur établissement sont conformes aux conventions collectives, puisqu'il a été démontré clairement que ces politiques existent parce qu'il est dans l'intérêt de l'organisation d'assurer la sécurité de ses patients ${ }^{2}$. Toutefois, dans le cas de St. Michael's Hospital v. Ontario Nurses' Association, l'arbitre a conclu qu'une exigence plus large de se faire vacciner ou de porter un masque (en dehors du contexte d'une éclosion) constituait un exercice déraisonnable du pouvoir de gestion, puisque les preuves que le port du masque par les travailleurs de la santé était un moyen efficace de protéger les patients contre la grippe étaient insuffisantes, inadéquates et entièrement non convaincantes ${ }^{7}$.

Ces 2 exemples montrent que le caractère raisonnable d'un mandat dépend de plusieurs facteurs contextuels. Dans le cas de la pandémie de COVID-19, compte tenu de l'effet protecteur conféré par l'utilisation appropriée d'équipement de protection individuelle (EPI) et les nouvelles données suggérant que le port du masque réduit la transmission du SRAS-CoV- $2^{8}$, la question est plutôt la suivante : un employeur peut-il raisonnablement exiger que ses employés se fassent vacciner même dans les cas où les employés disent qu'ils vont porter de l'EPI ou prendre d'autres précautions appropriées?

\section{Contestation de mandats du gouvernement en vertu de la Charte}

Le risque de contestation d'un mandat en vertu de la Charte est plus élevé pour les règles gouvernementales claires imposant le moment et l'endroit où les travailleurs de la santé devront être vaccinés que pour les mandats issus d'employeurs individuels. Cependant, la Charte peut permettre au gouvernement de défendre une politique de vaccination obligatoire des travailleurs de la santé, à condition que des exceptions appropriées soient prévues en cas de problèmes de santé sous-jacents ou pour les personnes qui s'opposent à la vaccination pour des raisons religieuses ou pour objection de conscience (le scepticisme concernant les bases scientifiques de la vaccination ne mènera probablement pas à un accommodement).

Soyons clairs : une règle gouvernementale imposant la vaccination ou le retrait des travailleurs de la santé qui interagissent directement avec les patients ne forcerait personne à se faire vacciner; la vaccination obligatoire imposée par le gouvernement serait hautement vulnérable à une contestation en vertu de la Charte. La règle imposerait plutôt des conséquences économiques aux travailleurs refusant la vaccination sans objection valide. À notre avis, une telle obligation - vaccination ou retrait du travail - ne constituerait pas un traitement médical forcé et ne violerait pas à première vue l'article 7 de la Charte, malgré le fardeau économique substantiel imposé aux travailleurs de la santé qui refuseraient de recevoir le vaccin. Jusqu'à maintenant, la Charte n'a pas été utilisée pour protéger les intérêts économiques, plus précisément le «droit au travail ». Si des contestataires réussissaient à faire valoir que l'obligation viole à première vue l'article 7 de la Charte, ils devront quand même démontrer que 
l'atteinte n'était pas en conformité avec les « principes de justice fondamentale ». Enfin, les gouvernements auront la possibilité de justifier l'obligation en argumentant qu'il s'agissait d'un moyen proportionnel en contexte de pandémie de COVID-19 (article 1).

\section{Les travailleurs de la santé peuvent-ils avancer que le port d'EPI suffit pour les protéger et protéger leurs patients?}

Le port d'EPI offre-t-il à lui seul une protection suffisante? La réponse influencera la probabilité de succès d'une contestation, que ce soit dans le contexte du droit du travail ou de la Charte.

Comme nous l'avons mentionné précédemment, bien que le moyen soit controversé, une province ou un employeur pourrait justifier une politique obligeant les travailleurs de la santé à choisir entre se faire vacciner contre le SRAS-CoV-2 ou accepter un retrait du travail sans salaire. Dans ce cas, un tribunal ou un arbitre en droit du travail devrait tenir compte des risques découlant de la pandémie de COVID-19, plus précisément des risques d'infection, de morbidité et de mortalité relativement élevés associés à la maladie, des risques accrus auxquels sont exposés les patients vulnérables, des risques propres aux travailleurs de la santé et des dangers généraux pour les patients si le personnel, déjà surchargé, est décimé par de nombreux cas de COVID-19. Pour être constitutionnellement acceptable, tout mandat devrait s'accompagner d'exemptions pour les personnes ayant des problèmes de santé sous-jacents rendant la vaccination impossible et celles ayant des objections de conscience ou des objections sincères sur des bases religieuses ${ }^{9}$. Après le début de la distribution des premiers vaccins, les travailleurs de la santé canadiens devront encore porter l'EPI approprié, même une fois qu'ils seront vaccinés. Les travailleurs de la santé pourraient-il alors exiger qu'on leur permette de porter de l'EPI (ou, potentiellement, un masque) au lieu de se faire vacciner? La probabilité de succès d'une contestation de la vaccination obligatoire en raison de l'efficacité suffisante de l'EPI employé seul sera basée sur plusieurs facteurs et pourrait dépendre de preuves non encore publiées.

Les différences dans les milieux de soins pourraient influencer les décisions : par exemple, dans une unité psychiatrique, le port d'EPI et les mesures de distanciation physique pourraient constituer une stratégie réaliste et suffisante pour assurer la sécurité des patients. Un autre facteur pertinent serait la présentation de données sur le coût de l'EPI et l'effort nécessaire pour s'en procurer, ainsi que, plus concrètement, son utilisation appropriée de manière à réduire efficacement le risque d'infection par le SRAS-CoV-2, pour les travailleurs de la santé et leurs patients. L'efficacité de différents vaccins hors du contexte des essais cliniques pourrait également entrer en ligne de compte (p. ex. : protection conférée aux personnes atteintes de certaines affections non étudiées dans le cadre des essais). Par ailleurs, comme l'argument juridique en faveur de la vaccination obligatoire repose en partie sur le désir de protéger les patients et travailleurs de la santé vulnérables, il faudra comparer son efficacité à celle de l'EPI pour ce qui est de la transmission du SRAS-CoV-2. Ici, les données ne permettent pas de trancher aussi clairement : nous n'avons pas encore accès aux résultats complets des essais randomisés et contrôlés sur la capacité les vaccins contre le SRAS-CoV-2 à prévenir la transmission du virus, mais on s'attend au moins à une certaine diminution de l'infectivité ${ }^{10,11}$. Le suivi réalisé dans le cadre des essais devrait fournir des données permettant de répondre à cette question cruciale. À notre avis, un gouvernement provincial pourrait invoquer le principe de précaution pour justifier la vaccination obligatoire des travailleurs de la santé sans preuve définitive de réduction de la transmission virale, compte tenu de la gravité particulière de la pandémie de COVID-19. En outre, la vaccination obligatoire pourrait au moins se justifier jusqu'à ce que la pandémie soit maîtrisée, puisqu'il est essentiel de maximiser la santé du personnel disponible pour traiter les patients atteints de la COVID-19. Si les données issues des essais de phase 3 indiquent une réduction de l'infectivité, cela ferait considérablement pencher la balance en faveur de la vaccination obligatoire, puisque de telles preuves n'existent pas pour l'EPI.

\section{Quelles autres considérations devraient être prises en compte?}

Puisque les données sur l'innocuité et l'efficacité de bon nombre de vaccins n'ont pas encore toutes été publiées, il se pourrait que certaines politiques se justifient pour certains vaccins contre le SRAS-CoV-2, mais pas pour d'autres, surtout si les profils d'efficacité et d'innocuité issus des essais et de la surveillance diffèrent considérablement. On pourrait donc se retrouver devant des situations où les travailleurs de la santé accepteraient une politique à condition que le vaccin administré ait un meilleur profil d'innocuité et d'efficacité, auquel l'employeur pourrait ne pas avoir accès.

L'existence de recours en cas de dommages découlant de politiques de vaccination obligatoire sera primordiale. Nous nous réjouissons de l'annonce récente d'un programme de soutien aux victimes d'une vaccination sans égard à la faute et attendons sa mise en place avec impatience ${ }^{12}$. Sans être une panacée, le programme offre une certaine sécurité aux travailleurs de la santé obligés de se faire vacciner pour accomplir leur travail.

\section{Conclusion}

Le sujet de la vaccination obligatoire est émotionnellement chargé. Même comparativement aux nombreux défis que pose la pandémie de COVID-19 sur les plans scientifique, juridique et politique, la question de la vaccination obligatoire est un véritable nœud gordien. L'administration d'un vaccin efficace aux travailleurs de la santé protège à la fois le personnel de la santé et les patients, ce qui réduit le fardeau global que fait peser la COVID-19 sur les services et garantit la présence d'un personnel suffisant pour répondre aux besoins de santé de la population durant la pandémie. Il 
est tout à fait possible qu'on puisse prouver que la vaccination réduit la transmission du SRAS-CoV-2 aux patients vulnérables.

Les gouvernements provinciaux et territoriaux devraient énoncer des règles claires encadrant la vaccination des travailleurs de la santé dans les milieux publics et privés au lieu d'en laisser la responsabilité aux employeurs. Bien qu'il soit possible que l'obligation pour les travailleurs de la santé de se faire vacciner ou de s'absenter du travail sans salaire soit contestée en vertu de la Charte, les gouvernements devraient être en mesure de défendre leur position avec succès. Pour être défendable, une telle obligation devra s'accompagner d'exemptions pour les personnes qui ne peuvent être vaccinées ou qui s'opposent à la vaccination pour des raisons religieuses ou pour objection de conscience justifiées. La possibilité qu'une personne étaie son opposition par l'argument que le port d'EPI compense l'absence de vaccination est un problème plus épineux : les gouvernements devraient évaluer avec soin et régulièrement les données probantes sur l'efficacité de l'EPI comparativement à l'efficacité démontrée des vaccins disponibles. Par ailleurs, le gouvernement devra financer et appuyer un système robuste de surveillance postcommercialisation des vaccins à l'échelle du pays, afin de mieux connaître l'efficacité des vaccins en contexte réel (p. ex. : sur des populations non incluses dans les essais), la gravité des cas et les effets indésirables, et financer d'autres recherches sur l'incidence des vaccins sur la transmission ${ }^{13}$. Ces données seront essentielles pour comprendre la capacité réelle des différents vaccins à protéger les travailleurs de la santé et les patients, ce qui permettra aux gouvernements provinciaux de calibrer leurs politiques de vaccination du personnel en conséquence.

\section{Références}

1. Ismail SJ, Zhao L, Tunis MC, et al.; National Advisory Committee on Immunization. Key populations for early COVID-19 immunization: preliminary guidance for policy. CMAJ 2020;192:E1620-32.

2. Gruben V, Reed AS, McGreer A. Health care workers, mandatory influenza vaccination policies and the law. CMAJ 2014;186:1076-80.

3. Petersen E, Koopmans M, Go U, et al. Comparing SARS-CoV-2 with SARS-CoV and influenza pandemics. Lancet Infect Dis 2020;20:e238-44.

4. Piroth L, Cottenet J, Mariet A-S, et al. Comparison of the characteristics, morbidity, and mortality of COVID-19 and seasonal influenza: a nationwide, population-based retrospective cohort study. Lancet Respir Med 2020 Dec. 17 [cyberpublication avant impression]. doi: 10.1016/S2213-2600(20)30527-0.

5. COVID-19 vaccines: no time for complacency. Lancet 2020;396:1607.

6. St. Peter's Health System v. Canada Union of Public Employees, Local 778 (Flu vaccination grievance) [2002] 106 L.A.C. (4th) 170.

7. St. Michael's Hospital v. Ontario Nurses'Association, CANLII 82519.
8. Brainard J, Jones NR, Lake IR, et al. Community use of face masks and similar barriers to prevent respiratory illness such as COVID-19: a rapid scoping review. Euro Surveill 2020;25:2000725.

9. Thomas B, Flood CM. Eliminating religious and philosophical exemptions: the next step in Ontario's campaign against vaccine hesitancy. Healthc Policy 2020;16:14-20.

10. Mandavilli A. Here's why vaccinated people still need to wear a mask. The New York Times 2020 Dec. 8, updated 2020 Dec. 9. Accessible ici : www.nytimes. com/2020/12/08/health/covid-vaccine-mask.html (consulté le 6 janv. 2021).

11. Vaccines and Related Biological Products Advisory Committee meeting presentation. mRNA-1273: sponsor briefing document addendum, Dec. 17, 2020. Silver Spring (MD): Food and Drug Administration; 2020. Accessible ici : www. fda.gov/media/144453/download (consulté le 5 janv. 2021).

12. Government of Canada Announces pan-Canadian vaccine injury support program [communiqué de presse]. Public Health Agency of Canada; modified 2020 Dec. 10. Accessible ici : www.canada.ca/en/public-health/news/2020/12/ government-of-canada-announces-pan-canadian-vaccine-injury-support -program.html (consulté le 11 janv. 2021).

13. Canadian National Vaccine Safety Network (CANVAS): What is CANVAS? Halifax: Canadian Immunization Research Network. Accessible ici : cirnetwork.ca/ network/national-ambulatory-network/ (consulté le 6 janv. 2021).

Intérêts concurrents : Kumanan Wilson est PDG de CANImmunize. Aucun autre intérêt concurrent n'a été déclaré.

Cet article a été révisé par des pairs.

Affiliations : Faculté de droit - Section de common law (Flood, Thomas), et Faculté de médecine - École d'épidémiologie et de santé publique (Wilson), Université d'Ottawa, Ottawa, Ont; Institut de recherche Bruyère (Wilson), Ottawa, Ont; Institut de recherche de l'Hôpital d'Ottawa (Wilson), Ottawa, Ont.

Collaborateurs : Tous les auteurs ont contribué à la conception du travail, ont rédigé le manuscrit et en ont révisé de façon critique le contenu intellectuel important; ils ont donné leur approbation finale pour la version destinée à être publiée et assument l'entière responsabilité de tous les aspects du travail.

Propriété intellectuelle du contenu : Il s'agit d'un article en libre accès distribué conformément aux modalités de la licence Creative Commons Attributions (CC BY-NC-ND 4.0), qui permet l'utilisation, la diffusion et la reproduction dans tout médium à la condition que la publication originale soit adéquatement citée, que l'utilisation se fasse à des fins non commerciales (c.-à-d. recherche ou formation) et qu'aucune modification ni adaptation n'y soit apportée. Pour plus de renseignements, consulter le https://creativecommons.org/licenses/ by-nc-nd/4.0/deed.fr.

Financement : Le financement pour cette recherche a été fourni en partie par le Groupe de travail sur l'immunité face à la COVID-19, créé par le gouvernement du Canada en collaboration avec les Instituts de recherche en santé du Canada.

Déclaration d'intérêts : Kumanan Wilson est marié à une employée du JAMC, qui n'a pas participé au processus ayant mené au choix de cet article.

Correspondance : Bryan Thomas, bryan.thomas@uottawa.ca 